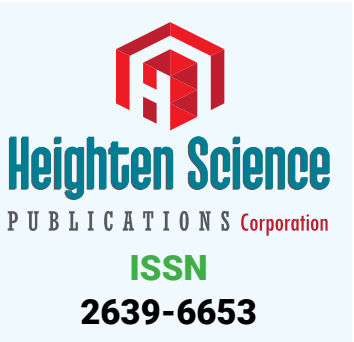

*Address for Correspondence: Henrik Rueffert, Department of Anaesthesiology, Intensive Care and Pain Therapy, Leipziger Str. 45, 04435 Schkeuditz, Germany, Tel. +49 34204808263 ; Fax.: +493420480 8245;

Email: Henrik.Rueffert@helios-gesundheit.de

Submitted: 21 September 2018

Approved: 16 October 2018

Published: 17 October 2018

Copyright: () 2018 Taubert M, et al. This is an open access article distributed under the Creative Commons Attribution License, which permits unrestricted use, distribution, and reproduction in any medium, provided the original work is properly cited

Check for updates

\title{
Challenges in the diagnosis and management of severe Pneumocystis jirovecii pneumonia in a non-HIV- infected patient - A case report
}

\author{
Mark Taubert', Lorenz Weidhase ${ }^{2}$, Sirak Petros ${ }^{2}$ and Henrik Rueffert ${ }^{1 *}$ \\ 'Department of Anaesthesiology, Intensive Care \& Pain Therapy, Helios Klinik Schkeuditz; Germany \\ ${ }^{2}$ Medical Intensive Care, University Hospital Leipzig, Germany
}

\section{Abstract}

A 64-year-old woman was referred to our hospital due to progressive dypnoea for the past week, combined with fever and type 1 respiratory failure. White blood cell count and procalcitonin level were normal. The Chest X-ray showed bilateral disseminated pulmonary infiltrates. Within the next 24 hours the patient developed a severe ARDS. A first diagnostic work-up for typical and atypical pathogens as well as serological tests for CMV, RSV, HIV and HSV were negative. Analysis of a second bronchoalveolar lavage fluid revealed Pneumocystis jiroveci DNA. The patient was successfully treated with trimethoprim-sulfamethoxazole and off label use with caspofungin. The cause of the infection was a six week treatment with dexamethasone. The patient developed a toxic epidermal necrolysis during further course, but completely recovered.

Pneumonia with Pneumocystis jirovecii must also be taken into account in non-HIV patients, whenever there are any indications that cellular immunity may be depressed.

\section{Introduction}

Pneumocystis jirovecii (formerly Pneumocystis carinii, PCP) results in a serious pulmonary infection in patients with acquired immunodeficiency syndrome, but it can also affect patients on immunosuppressive medication, transplant recipients or those with hematological or solid malignancies. The organism is currently placed phylogenetically in the fungal kingdom, but also shares biological characteristics with protozoa. It has a unique tropism for the alveolar epithelium and attaches but usually does not invade host cells.

Trimethoprim-sulfamethoxazole (TMP-SMZ) is the first-line treatment of PCP, while a combination of primaquine and clindamycin is the preferred alternative for treatment failures or intolerance. Echinocandins have also been shown to be effective in treating PCP. TMP-SMZ primarily eliminates trophic forms of Pneumocystis, whereas caspofungin clears cystic forms which might play a key role in transmission.

The mortality rate of PCP in non-HIV infected patients is high (35-50\%) compared to HIV patients (10-20\%) and the clinical outcome, independent of severity, is worse [1].

\section{Case Report}

A 64-year-old female was admitted to the emergency ward with progressive dyspnea, fever and fatigue. Four days prior to this presentation, she was discharged from another hospital, where she was investigated for chest pains. An acute coronary heart disease and pulmonary arterial embolism were excluded. 
Initial medical examination revealed mild cardiovascular distress and impaired consciousness, with a body temperature of $39.0^{\circ} \mathrm{C}$ and type 1 respiratory failure $\left(\mathrm{SpO}_{2}\right.$ 85\%; $61 \mathrm{O}_{2}$ per minute). Chest X-ray showed bilateral disseminated infiltrates (Figure 1A). White blood cell count (6.9 Gpt/l) and procalcitonin $(0.29 \mu \mathrm{g} / \mathrm{l})$ were normal; while C-reactive protein level was increased $(295 \mathrm{mg} / \mathrm{l})$.

Due to the partial respiratory insufficiency $\left(\mathrm{PaO}_{2} 47.8 \mathrm{mmHg}\right)$ non-invasive positive pressure ventilation was started $\left(\mathrm{FiO}_{2} 60 \%, \mathrm{p}_{\max } 14 \mathrm{mmHg}\right.$, PEEP $\left.6 \mathrm{mmHg}\right)$.

Blood samples and bronchoalveolar lavage fluid were collected for microbiological analysis and calculated antimicrobial therapy with ceftriaxone (one dose of $2 \mathrm{~g}$ i.v.) and clarithromycin (two doses of $500 \mathrm{mg}$ i.v. per day) was initiated. Influenza A and B virus RNA screening was negative.

Despite goal-directed volume, vasopressor support and antibiotic therapy, the patient's condition showed a rapid deterioration; 24 hours after admission orotracheal intubation and invasive ventilation had to be started. The $\mathrm{PaO}_{2} / \mathrm{FiO}_{2}$ ratio was 60 , corresponding to a severe ARDS [2] (Figure 1B).

Prone positioning for 12 hours per day was started immediately. Tidal volume was limited to $6 \mathrm{ml} / \mathrm{kg}$ per ideal body weight, combined with protocol-based setting of driving pressure values $\Delta \mathrm{P} \leq 15 \mathrm{mmHg}$. In due course, higher ventilator pressures $\left(\mathrm{p}_{\max }\right.$ $30 \mathrm{mmHg}, \mathrm{p}_{\text {mean }} 20 \mathrm{mmHg}$, PEEP 14-19 mmHg) were necessary. Because of perioral rash with blisters, a pulmonary herpes infection was suspected and a continuous course of acyclovir (15 mg/kg body mass) was started. Diagnostic work-up for mycoplasma pneumoniae, chlamydophila pneumoniae and legionella spp. as well as serological search for CMV, RSV, HIV and HSV was negative.

$\mathrm{FiO}_{2}$ couldn`t be reduced below 0.65 . A repeat bronchoscopy showed an alveolar haemorrhagic syndrome (Figure 1C) and the calculated antimicrobial therapy was extended to meropenem (three doses of $1 \mathrm{~g}$ i.v. per day). Microbiological analysis of the bronchoalveolar lavage fluid revealed the presence of HSV as well as Pneumocystis jiroveci DNA.

Antibacterial therapy was then changed to TMP-SMZ (four dosages of $2400 \mathrm{mg}$ TMP-SMZ per day within $650 \mathrm{ml}$ glucose $5 \%$ ). Due to the severity of ARDS, we chose an off-label use combination with caspofungin for the next 7 days.

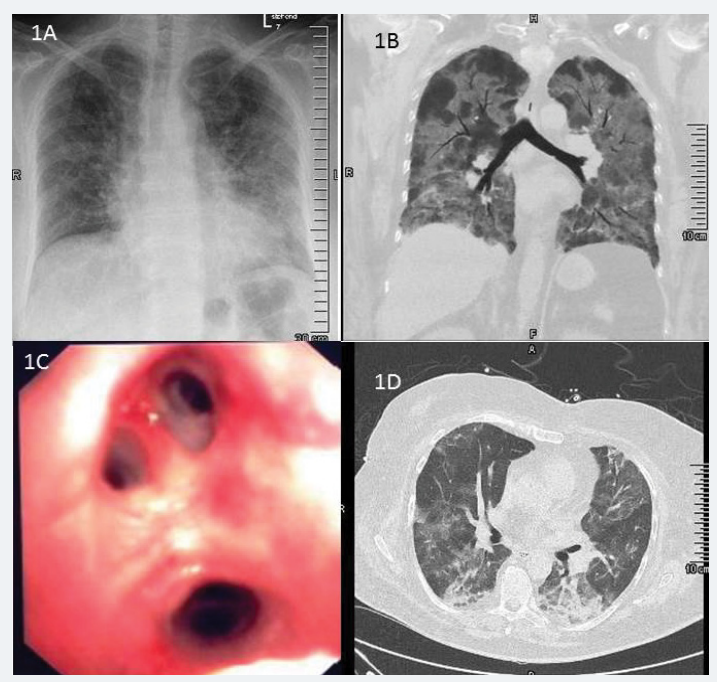

Figure 1: A) Chest X-ray showed bilateral disseminated infiltrates, mainly in in middle and basal regions of the lungs. B) Thoracic computed tomography (CT) showing bilateral lung infiltrates. Cardiac failure and fluid overload were excluded by transthoracic echocardiography. C) Bronchoscopy on day 3 which shows an alveolar haemorrhagic syndrome. D) Thoracic CT scan, day 14 , shows pulmonary improvement. Dense consolidation significantly absorbed. 
Detailed history taking (patient's relatives and general practitioners) revealed a six week course of dexamethasone (single dose of $16 \mathrm{mg}$ p.o. per day; two weeks in combination with ibuprofen $1200 \mathrm{mg}$ per day) for lumbar intervertebral disc herniation before onset of disease.

Besides continuous renal replacement therapy, daily prone positioning (12 hours) was continued for 6 days and aerosolized prostaglandine was administered for 5 days. Percutaneous tracheostomy was performed on day 5. Substantial improvement of respiratory and renal function was observed only from day 12 onwards (Figure 1D), when periods of spontaneous breathing could be extended to 12 hours and the CRRT stopped. On day 16, the patient developed a severe cutaneous and mucous adverse reaction (Figure. 2) affecting more than $20 \%$ of the body surface. This was considered as toxic epidermal necrolysis (TEN), most probably caused by cotrimoxazole. The TMP-SMZ therapy was immediately stopped and after skin biopsy confirmed TEN, antimicrobial therapy was switched to a combination of primaquine and clindamycin. This antibiotic treatment could finally be stopped on day 23 , when the tracheostoma was also removed after sufficient unassisted spontaneous breathing was achieved. The epidermal lesions subsided with local wound management. The patient was transferred to the general ward on day 24 and discharged home on day 41 .

\section{Discussion}

The presented case was a challenge in many ways. Firstly, there was a severe ARDS with its predicted hospital mortality of almost $50 \%$. Prone position, consequent lung protective ventilation and avoiding fluid overload are proven strategies in ARDS therapy. The driving pressure (within a range of $\leq 15 \mathrm{mmHg}$ ) has recently become a valid outcome parameter of ARDS (15). Potential intra-pulmonary shunt may be reduced by aerosolized prostaglandine treatment. However, all these strategies are useless if the basic cause of ARDS remains undiscovered. The detection of $P$. jirovecii was very surprising as because there were initially no indications for any immunocompromised conditions. Detailed history taking revealed the inappropriate treatment of lumbar intervertebral disc herniation with high dose dexamethasone. The mortality of $P$. jirovecii in non-HIV patients is extremely high (50\%). High dose TMP-SMZ is the treatment of choice but requires large amount of fluid for parenteral administration. Serious adverse effects such as TEN (mortality rate 25 to $70 \%$ ) may be observed [4]. We combined the antimicrobial TMP-SMZ therapy with caspofungin right from the start. The use of caspofungin is still controversial in human PCP, but both combination with TMP-SMZ and clindamycin can be taken into account as optional savage therapy [5].

\section{Acknowledgement}

The authors would like to thank Dr. C. Coetzee for revision of content and spelling.

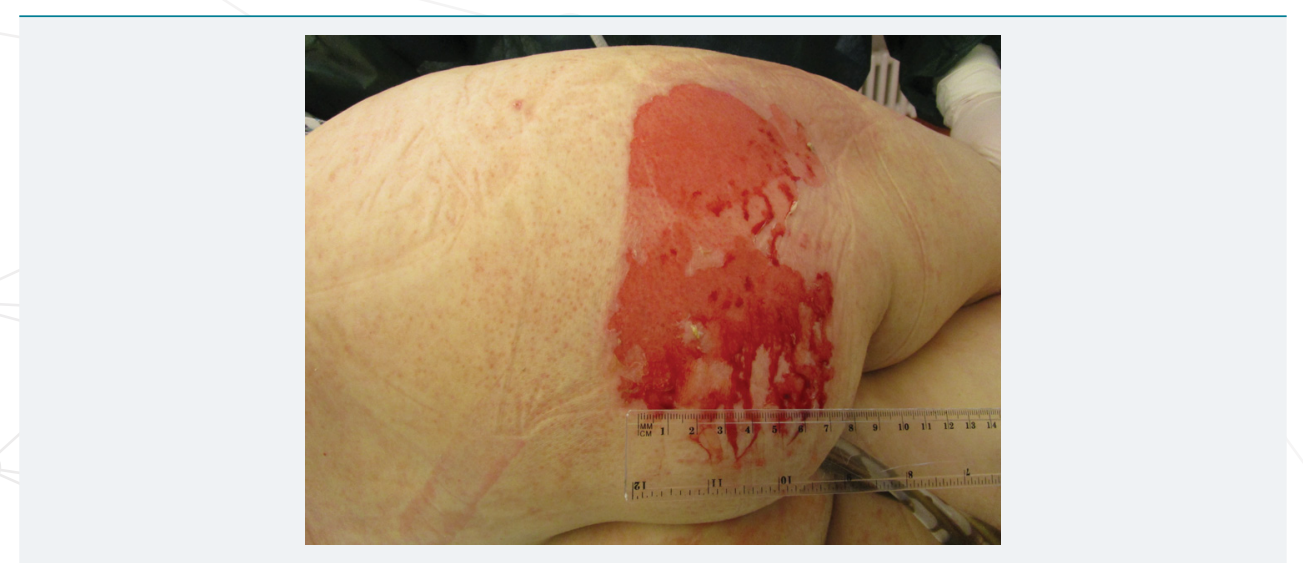

Figure 2: Dusky epidermal lesions, primarily affecting the gluteal region, but then partially spread over the trunk, oral and bronchial mucosa. Toxic epidermolysis (TEN) was confirmed by skin biopsy. 


\section{Disclosure statement}

Appropriate written informed consent was obtained for publication of this case and images. Ethical approval to report this case was not required.

\section{References}

1. Mansharamani NG, Garland R, Delaney D, Koziel H. Management and outcome patterns for adult Pneumocystis carinii pneumonia, 1985 to 1995: comparison of HIV-associated cases to other immunocompromised states. Chest. 2000; 118: 704-711. Ref.: https://goo.gl/EPe8B7

2. Bellani G, Laffey JG, Pham T, Fan E, Brochard L, et al. Epidemiology, Patterns of Care, and Mortality for Patients With Acute Respiratory Distress Syndrome in Intensive Care Units in 50 Countries. JAMA. 2016; 315: 788-800. Ref.: https://goo.gl/LMG3Dv

3. Lobo ML, Esteves F, de Sousa B, Cardoso F, Cushion MT, et al. Therapeutic Potential of Caspofungin Combined with Trimethoprim-Sulfamethoxazole for Pneumocystis Pneumonia: A Pilot Study in Mice. PLoS One., 2013; 8: e70619. Ref.: https://goo.gl/N9annU

4. Sekula P, Dunant A, Mockenhaupt M, Naldi L, Bouwes Bavinck JN, et al. Comprehensive survival analysis of a cohort of patients with Stevens-Johnson syndrome and toxic epidermal necrolysis. J. Invest. Dermatol. 2013; 133: 1197-204. Ref.: https://goo.gl/JRc6T8

5. $\mathrm{Li} \mathrm{H}$, Huang $\mathrm{H}, \mathrm{He} \mathrm{H}$. Successful treatment of severe Pneumocystis pneumonia in an immunosuppressed patient using caspofungin combined with clindamycin: a case report and literature review. BMC. Pulm. Med. 2016; 16: 144. Ref.: https://goo.gl/wuvPtt 